\title{
Relations entre la prise alimentaire et la consommation d'oxygène des organes drainés par la veine porte chez le porc éveillé
}

\author{
A Rérat, $P$ Vaissade
}

INRA, unité Absorption intestinale et Métabolisme hépatique, 78352 Jouy-en-Josas cedex, France

(Reçu le 2 décembre 1992; accepté le 1er avril1993)

\begin{abstract}
Résumé - L'absorption intestinale de glucose et d'azote alpha aminé, l'apparition d'acides $D$ et Llactiques dans la veine porte et la consommation d'oxygène des organes de l'aire abdominale splanchnique extra-hépatique ont été étudiées simultanément chez des porcs, soit au cours de la période post-prandiale, soit au cours d'une période correspondante de maintien au jeûne. Dans ce but, 5 porcs en croissance (poids vif : $59,4 \pm 3,5 \mathrm{~kg}$ ) ont été munis de cathéters permanents dans la veine porte et l'artère carotide et d'une sonde débitmétrique autour de la veine porte, dispositif permettant de mesurer les échanges entre sang et lumière intestinale. Ils ont reçu durant une semaine un régime semisynthétique contenant $6 \%$ de cellulose purifiée (R6) puis, la semaine suivante, un régime à $16 \%$ de cellulose purifiée (R16), l'ordre de cette distribution étant alterné d'un animal à l'autre. Durant chacune de ces semaines, ils ont été, après $16 \mathrm{~h}$ de jeûne, soumis à 2 tests successifs de $5 \mathrm{~h}$, soit après ingestion d'un repas de $800 \mathrm{~g}$ (R 6 ou R16), soit sans ingestion de ce repas (J6 ou J16). Des prélèvements sanguins ont été pratiqués à intervalles de 15 à $30 \mathrm{~min}$ pendant ces tests et les analyses ont porté sur l'oxygène à l'aide d'un appareil Radiométer ABL3 et sur les divers nutriments et métabolites cités. Dans ces conditions, la consommation d'oxygène est plus élevée $(P<0,01)$ durant la période postprandiale $(5,41 \pm 0,19 \mathrm{mmol} / \mathrm{kg} / \mathrm{h})$ que durant le maintien du jeûne $(4,64 \pm 0,19 \mathrm{mmo} / \mathrm{kg} / \mathrm{h})$, alors que la disponibilité de l'oxygène n'est que faiblement augmentée $(P<0,05)(14,01 \pm 0,66$ vs $13,08 \pm 0,76)$. Par contre, le coefficient d'extraction de l'oxygène n'est pas significativement augmenté par la prise alimentaire. L'apparition d'acide L-lactique dans le sang portal est plus élevée $(P<0,001)$ pendant la période post-prandiale $(3,83 \pm 0,32 \mathrm{~g} / \mathrm{h})$ que pendant la période de maintien du jeûne $(1,23 \pm 0,23 \mathrm{~g} / \mathrm{h})$. La dépense énergétique due au métabolisme aérobie des organes drainés par la veine porte se situe entre 35,7 et $37,8 \mathrm{kcal} / \mathrm{h}$ après l'ingestion d'un repas, soit 11 à $18 \%$ de plus que lors du maintien du jeûne. Le métabolisme anaérobie ne représente que $0,3-0,4 \%$ (à jeun) et $1,1-1,3 \%$ (période postprandiale) de ce métabolisme aérobie. La consommation d'oxygène n'est pas modifiée par la teneur en cellulose du régime.
\end{abstract}

jeun / prise alimentaire / consommation d'oxygène / organes drainés par la veine porte / porc

Summary - Influence of feeding on oxygen consumption by the portal vein-drained organs in the conscious pig. The intestinal absorption of glucose and $x$-amino nitrogen, the appearance of $D$-and $L$-lactic acids in the portal vein and the consumption of oxygen by the organs of the abdominal extrahepatic splanchnic area were studied simultaneously in pigs either during the postprandial period or during a fasting period of the same length. For this purpose, 5 growing pigs $(59.4 \pm 3.5 \mathrm{~kg}$ live weight) were fitted with permanent catheters in the portal vein and carotid artery and with an electromagnetic flowmeter probe around the portal vein. This device was used to measure the exchanges between the blood and the intestinal lumen. For a period of $1 \mathrm{wk}$, the animal received a semisynthetic diet containing $6 \%$ purified cellulose $(R 6)$ and then during the next $w k$, a diet containing 
$16 \%$ purified cellulose (R16), the order of distribution being alternated from one animal to the next. During each of these $2 \mathrm{wk}$ and after a fasting period of $16 \mathrm{~h}$, they were subjected to 2 successive tests of $5 \mathrm{~h}$, either after intake of an 800-g meal (R) (R6 or R16), or after no meal intake (J6 or J16). During these tests, blood samplings were made at 15 to 30-min intervals, the consumption of oxygen was recorded by an $A B L 3$ radiometer and the afore mentioned nutrients and metabolites analysed in these samples. The consumption of oxygen was higher $(\mathrm{P}>0.01)$ during the postprandial period $(5.41 \pm 0.19 \mathrm{mmol} / \mathrm{kg} / \mathrm{h})$ than during the fasting period $(4.64 \pm 0.19 \mathrm{mmol} / \mathrm{kg} / \mathrm{h})$ while the availability of oxygen only increased slightly $(\mathrm{P}<0.05)(14.01 \pm 0.66 \mathrm{vs} 13.08 \pm 0.76 \mathrm{mmol} / \mathrm{kg} / \mathrm{h})$. By contrast, the oxygen extraction coefficient did not significantly increase with meal intake. The appearance of $L$ lactic acid in the portal blood was higher $(P<0.001)$ during the postprandial period $(3.83 \pm 0.32 \mathrm{~g} / \mathrm{h})$ than during the fasting period $(1.23 \pm 0.23 \mathrm{~g} / \mathrm{h})$. The energy expenditure due to the aerobic metabolism of the organs drained by the portal vein ranged from 35.7 to $37.8 \mathrm{kcal} / \mathrm{h}$ after meal intake, ie 1$18 \%$ more than after fasting. The anaerobic metabolism only represented $0.3-0.4 \%$ (fasting) and $1.1-$ $1.3 \%$ (postprandial period) of this aerobic metabolism. Oxygen consumption was not changed by the dietary cellulose content.

\section{fasting / feeding / oxygen consumption / portal vein-drained organs / pig}

\section{INTRODUCTION}

La prise d'aliment est à l'origine de divers phénomènes au niveau du tractus digestif, phénomènes qui intéressent la motricité gastro-intestinale, les sécrétions exocrines et endocrines et l'absorption. II en résulte une augmentation de l'activité des organes digestifs, qui s'accompagne d'une demande accrue d'oxygène de leur part. Ce besoin supplémentaire d'oxygène peut être couvert à la fois par une hyperémie post-prandiale régionale (Rérat et al, 1985 ) et par une extraction tissulaire plus élevée de l'oxygène artériel (Granger et Norris, 1980). Chez le porcelet nouveauné, ont ainsi été constatées une consommation d'oxygène accrue et une hyperémie de la région gastro-intestinale à la suite de l'ingestion des repas (Nowicki et al, 1983) et ces faits ont été confirmés récemment par Yen et al (1989) chez des porcs plus âgés (4 mois).

La présente expérience visait à déterminer l'influence de l'ingestion du repas et de la teneur cellulosique du régime sur la consommation d'oxygène des organes drainés par la veine porte, chez le porc non anesthésié. Une courte communica- tion a fourni quelques données préliminaires (Vaugelade et Rérat, 1991).

\section{MATÉRIEL ET MÉTHODES}

\section{Animaux}

Cinq porcs mâles castrés de race Large White, d'un poids vif de $40 \mathrm{~kg}$ environ, provenant du troupeau du département de nutrition de l'Institut national de la recherche agronomique (INRA, La Minière) ont été utilisés. Pendant un mois avant la mise en expérience, ils ont reçu un régime pré-expérimental bien équilibré, 2 fois/j (à $9 \mathrm{~h} 00$ et $17 \mathrm{~h} \mathrm{00)}$ à raison de $800-1000 \mathrm{~g} /$ repas. Le gain de poids durant cette période dépassait $600 \mathrm{~g} / \mathrm{j}$. À la période de la mise en expérience, leur poids moyen était de $59,4 \pm 3,5 \mathrm{~kg}$. Chaque animal a été muni sous anesthésie, d'une part, d'une sonde de débitmètre électromagnétique autour de la veine porte pour mesurer le débit de sang dans ce vaisseau, d'autre part, de 2 cathèters permanents, I'un placé dans la veine porte, l'autre dans le tronc brachiocéphalique par la voie carotidienne (Rérat et al, 1980). Les cathéters permettent de pratiquer des prélèvements sanguins indolores au cours des périodes expérimentales. Les animaux ont été placés dans des cages à contention et entretenus selon les principes d'éthique prescrits pour les animaux de laboratoire. Pendant la période pos- 
topératoires, ils ont reçu pendant $3 \mathrm{j}$ une dose journalière de $1200000 \mathrm{UI}$ de pénicilline et $1 \mathrm{~g}$ de streptomycine.

\section{Régimes}

Au cours de la période postopératoire, les animaux ont reçu en séquence 2 régimes expérimentaux (tableau I) administrés chacun pendant 8 à $10 \mathrm{j}$, l'ordre de cette distribution étant alterné d'un animal à l'autre. L'un de ces régimes (R6) contenait un taux modéré de cellulose purifiée $(6 \%)$, l'autre (R16) en contenait un taux élevé $(16 \%)$. Ces 2 régimes avaient des teneurs très voisines en énergie brute et en matières azotées totales. Au cours de la première semaine après l'opération chirurgicale, chaque animal a été ainsi progressivement accoutumé à l'un de ces 2 régimes, qu'il a consommé selon une échelle croissante passant de $100 \mathrm{~g}$ pour le pre-

Tableau 1. Composition centésimale des régimes.

$R 6$

$R 16$

\begin{tabular}{|c|c|c|}
\hline \multicolumn{3}{|l|}{ Régimes (\%) } \\
\hline Amidon de mais & 62,0 & 52,0 \\
\hline Farine de Poisson & 18,0 & 18,0 \\
\hline Cellulose pure & 6,0 & 16,0 \\
\hline Saccharose & 5,0 & 5,0 \\
\hline Huile d'arachide & 5,0 & 5,0 \\
\hline Concentré minéral & 3,0 & 3,0 \\
\hline Concentré vitaminique & 1,0 & 1,0 \\
\hline \multicolumn{3}{|c|}{ Caractéristiques nutritionnelles } \\
\hline Matière sèche (MS \%) & 91,33 & 91,81 \\
\hline Azote (\% MS) & 2,15 & 2,22 \\
\hline Cellulose (\% MS) & 5,40 & 13,90 \\
\hline Énergie brute & & \\
\hline $\begin{array}{l}\text { (kcal/g MS) } \\
\text { Énergie digestible }\end{array}$ & 4,398 & 4,369 \\
\hline (kcal/g MS) & 3,914 & 3,753 \\
\hline $\begin{array}{l}\text { Coefficient apparent } \\
\text { d'utilisation digestive }\end{array}$ & & \\
\hline de l'azo & 82,7 & 81,1 \\
\hline
\end{tabular}

a D'après Giusi-Périer et al (1989). mier jour, par étapes de $100 \mathrm{~g}$ par repas 2 fois/j (à $9 \mathrm{~h} 00$ et $17 \mathrm{~h} \mathrm{00}$ ) pour atteindre $800 \mathrm{~g}$ le cinquième jour. Une semaine après l'opération, il a recouvré une croissance de $600 \mathrm{à} 700 \mathrm{~g} / \mathrm{j}$, et a dès lors été soumis à 2 périodes d'épreuve de $5 \mathrm{~h}$, se déroulant à $24 \mathrm{~h}$ d'intervalle; ces 2 tests différaient par la distribution ou l'absence de distribution du repas matinal $(800 \mathrm{~g})$, le repas vespéral $(800 \mathrm{~g})$ étant maintenu le jour précédent. Puis il a été accoutumé au deuxième régime expérimental pendant une semaine, les épreuves étant faites dans les mêmes conditions que pour le premier régime. Ainsi, chaque animal a été soumis successivement à 2 fois 2 tests quotidiens, soit durant la période post-prandiale après ingestion d'un repas de $800 \mathrm{~g}$ d'un régime à $6 \%(R 6)$ ou à $16 \%$ (R16) de cellulose, soit durant une période correspondante de maintien du jeûne ( $\mathrm{J} 6$ et J16); dans tous les cas, il a reçu la veille à $17 \mathrm{~h} 00$ un dernier repas du régime étudié.

\section{Mesures}

Au cours de chaque période d'épreuve, on a procédé à l'enregistrement continu du débit sanguin dans la veine porte commençant 15 min avant l'heure du repas $\left(\mathrm{t}_{\mathrm{O}}\right)$ ou du début de la période d'étude du jeûne, et se terminant $5 \mathrm{~h}$ après; le calcul du débit se faisait par intégration des pointages manuels réalisés toutes les 5 min sur l'enregistrement graphique. Durant la même période, des prélèvements sanguins $(4 \mathrm{ml})$ ont été effectués dans la veine porte et l'artère carotide à $t_{0}-15, t_{0}$, puis à intervalles de $15 \mathrm{~min}$ jusqu'à la $2^{e} \mathrm{~h}$ et de $30 \mathrm{~min}$ jusqu'à la $5^{e} \mathrm{~h}$. Les pertes sanguines ont été compensées par l'injection de volumes adéquats de sérum sale $(\mathrm{NaCl}: 9 \mathrm{~g} / \mathrm{l})$. Une fraction (2 $\mathrm{ml})$ de chaque échantillon a été immédiatement utilisée pour la mesure des concentrations sanguines d'oxygène total à l'aide d'un analyseur de gaz sanguin Radiometer ABL 3 en tenant compte de la température et de la concentration en hémoglobine. Le reste de chaque échantillon $(2 \mathrm{ml})$ a été utilisé pour l'analyse du glucose (technique à la glucose oxydase : Hill et Kessler, 1961), de l'azote aminé (technique au trinitrobenzène sulfonate; Palmer et Peters, 1965 ) et des acides L-lactique (Minaire et al, 1965) et D-Lactique (Gawehn et Bergmeyer, 1974). 


\section{CALCULS ET MÉTHODES STATISTIQUES}

La méthode utilisée permet de quantifier les différences porto-artérielles, à intervalles de temps réguliers après le repas, que ces différences soient positives ou négatives. Ainsi, la quantité de nutriments ou de métabolites apparaissant dans la veine porte au cours de la période postprandiale peut être calculée par les formules suivantes:

$$
q=(C p-C a) F \mathrm{~d} t \text { et } Q=\sum_{t_{0}}^{t_{1}} q
$$

dans lesquelles $q$ est la quantité absorbée durant le temps court (5 min) pour lequel chaque facteur peut être considéré comme constant, $C p$ est la concentration portale des nutriments, $\mathrm{Ca}$ leur concentration artérielle, $F$ le débit de sang dans la veine porte et $Q$ la quantité de nutriments qui apparaissent par tranche horaire ou au cours de la période post-prandiale entre les temps $t_{1}$ et $t_{0}$.

Cette formule, appliquée à l'oxygène du sang, fournit les paramètres suivants :

- consommation d'oxygène : $V\left(\mathrm{O}_{2}\right)=\left[A\left(\mathrm{O}_{2}\right)-P\right.$ $\left.\left(\mathrm{O}_{2}\right)\right] F$;

- quantité d'oxygène disponible $D\left(\mathrm{O}_{2}\right)=A$ $\left(\mathrm{O}_{2}\right) \times F$;

- coefficient d'extraction de l'oxygène

$$
C U\left(\mathrm{O}_{2}\right)=\frac{V\left(\mathrm{O}_{2}\right)}{D\left(\mathrm{O}_{2}\right)} \times 100
$$

où $A\left(\mathrm{O}_{2}\right)$ et $P\left(\mathrm{O}_{2}\right)$ représentent les concentrations d'oxygène total dans les sangs artériel et portal, respectivement.

Les résultats sont exprimés sous forme de moyennes et écarts types à la moyenne, et les analyses statistiques (Snedecor et Cochran, 1967) utilisent la comparaison de 2 groupes de données par le test $t$ apparié de Student, ainsi que l'analyse de variance à 2 voies. Les données sont comparées pour chaque intervalle horaire après le début de la période d'observation et/ou sur l'ensemble de la période (données cumulées). Lorsque l'analyse de variance met en évidence l'effet significatif de l'état nutritionnel (jeûne vs repas) ou du taux de cellulose $(6 \%$ vs
$16 \%$ ), et qu'il n'existe aucune interaction entre ces 2 facteurs de variation, les données correspondantes (exemple J6 + J16 vs R6 + R16) sont regroupées et comparées dans les tableaux et graphiques.

\section{RÉSULTATS}

\section{Débit sanguin}

Le débit moyen dans la veine porte, pendant l'ensemble de la période d'observation de $5 \mathrm{~h}$, est analogue, que les animaux soient nourris ou à jeun, quel que soit le régime (tableau II). Les données correspondant à l'évolution par quart d'heure ont été regroupées pour comparer les animaux à jeun $(\mathrm{J} 6+\mathrm{J} 16)$ aux mêmes animaux nourris (R6 + R16) (fig 1). La prise de repas s'est traduite par une augmentation non significative $(P<0,06 ; n=10)$ de $4,5 \%$ du débit portal de $30 \mathrm{~min}$ après le repas, cette augmentation se maintenant de façon fluctuante à ce niveau pendant 2

Tableau II. Débit moyen de sang dans la veine porte au cours de la période post-prandiale $(5 \mathrm{~h})$ ou du maintien du jeûne, et variations selon le

\begin{tabular}{|c|c|c|c|}
\hline & $\begin{array}{l}\text { Poids vif } \\
\text { (kg) }\end{array}$ & $\begin{array}{l}\text { Débit de sa } \\
(\mathrm{ml} / \mathrm{min})\end{array}$ & $\begin{array}{l}g \text { portal } \\
(\mathrm{ml} / \mathrm{kg} / \mathrm{min})\end{array}$ \\
\hline $\mathrm{J} 6^{\circ}$ & $63,4 \pm 3,5$ & $2581 \pm 46$ & $41,2 \pm 2,2$ \\
\hline $\mathrm{R} 6^{\circ}$ & $62,2 \pm 3,6$ & $2548 \pm 59$ & $41,5 \pm 2,4$ \\
\hline $\mathrm{J} 16^{\circ}$ & $63,4 \pm 4,5$ & $2509 \pm 141$ & $40,0 \pm 2,6$ \\
\hline $\mathrm{R} 16^{*}$ & $61,6 \pm 4,4$ & $2559 \pm 134$ & $41,7 \pm 1,0$ \\
\hline
\end{tabular}
régime.

\footnotetext{
* R6 : période après ingestion d'un repas (régime à 6\% de cellulose); R16 : période après ingestion d'un repas (régime à $16 \%$ de cellulose); J6: période de maintien du jeûne après accoutumance au régime R6; J16: période de maintien du jeûne après accoutumance au régime R16.
} 


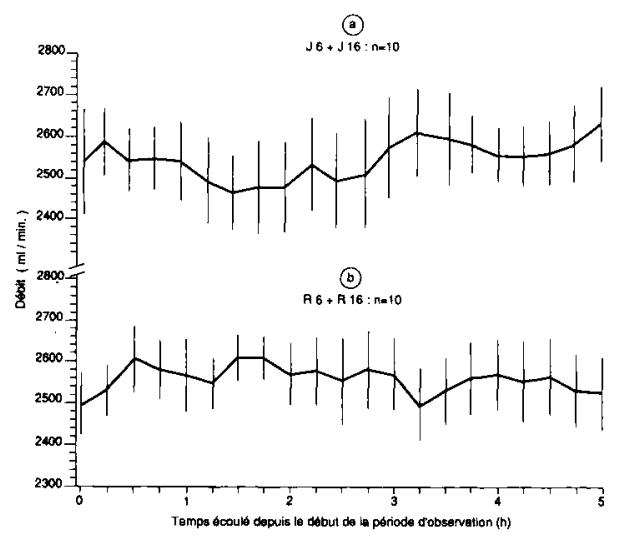

Fig 1. Évolution du débit sanguin dans la veine porte. a). Durant une période de maintien du jeûne débutant $16 \mathrm{~h}$ après le dernier repas $(800$ g). b). Durant la période post-prandiale après ingestion d'un repas de $800 \mathrm{~g}$. Chaque point représente la moyenne (les barres représentent l'écart type de la moyenne) de 10 données recueillies chez des animaux soumis au régime R6 (à taux modéré de cellulose, $n=5$ ) et au régime R16 (à taux élevé de cellulose, $n=5$ ) ou au jeûne correspondant (J6, $n=5$; J16, $n=5$ ).

à $3 \mathrm{~h}$ après le repas. Lors du maintien au jeûne, on n'enregistre pendant la même période que des variations très faibles du débit $( \pm 2 \%$ du débit initial selon le temps écoulé depuis le début de la période d'observation). Quel que soit l'état nutritionnel (jeûne vs repas), les variations interindividus sont très marquées, ce qui se traduit par des écarts types très élevés, et il n'existe aucune différence significative entre les 2 groupes d'observations, quelle que soit la durée de la période écoulée.

\section{Apparition de nutriments et métabolites dans la veine porte}

Les quantités de glucose apparues dans la veine porte sont rapportées dans le tableau III. II se produit une absorption faible ou modérée de glucose chez les animaux maintenus à jeun. Cette absorption, liée à la digestion du reliquat du repas de la veille correspondant à $20,4 \% \quad(\mathrm{~J} 6)$ et $14,1 \%$ (J16) de celui-ci, n'est pas significativement différente entre les animaux accoutumés au régime à $6 \%$ de cellulose et ceux accoutumés au régime à $16 \%$ de cellulose. Les quantités totales (en $5 \mathrm{~h}$ ) de glucose apparues dans la veine porte sont beaucoup plus importantes après ingestion du repas que lors du maintien du jeûne (R6 vs J6: $P<0,01 ; \mathrm{R} 16$ vs J16: $P<$ $0,05)$, ce qui reflète des différences horaires significatives (R6 vs J6:P<0,05 à 0,01 selon l'heure; R16 vs J16: $P<0,05$, sauf à la fre h). Cet effet de l'état nutritionnel est confirmé lorsque sont comparés les animaux à jeun ( $\mathrm{J} 6+\mathrm{J} 16)$ aux animaux nourris (R6 + R16) : les différences sont très hautement significatives $(P<0,01$ à $0,001)$. Les quantités de glucose absorbées sont plus élevées après ingestion du régime à $6 \%$ de cellulose qu'après ingestion du régime à $16 \%$ de cellulose, à la 4e h $(P<0,01)$ et sur la période totale $(P<$ $0,05)$. Quel que soit le régime, l'absorption horaire la plus élevée se situe à la $2^{e} \mathrm{~h}$ après le repas. En raison de l'apport plus faible de substances amylacées dans le régime à $16 \%$ de cellulose, le coefficient d'absorption du glucose (pourcentage des quantités apparues dans la veine porte par rapport aux quantités de glucose ingérées) n'est que légèrement supérieur (NS) après ingestion du régime à $6 \%$ de cellulose à ce qui se produit après ingestion du régime à $16 \%$ de cellulose, tant au plan horaire qu'au plan cumulé (R6: $51,6 \%$; R16 : $44,5 \%$ ).

Les quantités d'azote aminé apparues dans le sang portal (tableau IV) lors du maintien au jeûne sont faibles, correspondant à $33,7 \%(\mathrm{~J} 6)$ et $22,6 \%(\mathrm{~J} 16)$ des quantités ingérées lors du repas de la veille. Les quantités d'azote aminé absorbées durant la période de jeûne ont tendance à être plus élevées dans le cas du 
Tableau III. Évolution des quantités de glucose (en g) apparaissant dans le sang portal au cours de la période post-prandiale $(5 \mathrm{~h})$ ou du maintien du jeûne selon le régime ${ }^{1,2}$.

\begin{tabular}{|c|c|c|c|c|c|c|}
\hline & & rvalles de ten & os depuis le & b́but de la pét & de d'observ & (h) \\
\hline & $0-1$ & $1-2$ & $2-3$ & $3-4$ & $4-5$ & $0-5$ \\
\hline J6 1 & $37,0 \pm 10,6$ & $29,6 \pm 10,3$ & $23,9 \pm 7,3$ & $16,3 \pm 7,9$ & $14,3 \pm 7,6$ & $121,2 \pm 41,9$ \\
\hline $\mathrm{R} 6^{1}$ & $70,3 \pm 7,8$ & $72,8 \pm 8,8$ & $51,8 \pm 6,7$ & $62,7 \pm 1,2^{\mathrm{a}}$ & $49,3 \pm 6,2$ & $306,9 \pm 14,7^{A}$ \\
\hline$J 16^{1}$ & $23,6 \pm 7,5$ & $15,4 \pm 5,1$ & $13,7 \pm 5,9$ & $13,0 \pm 6,5$ & $8,2 \pm 5,4$ & $73,9 \pm 29,2$ \\
\hline R16 ${ }^{1}$ & $58,2 \pm 7,7$ & $60,4 \pm 6,7$ & $39,5 \pm 3,6$ & $39,0 \pm 4,0^{\circ}$ & $35,9 \pm 4,2$ & $233,1 \pm 32,7^{B}$ \\
\hline$J 6+J 16$ & $30,3 \pm 6,5$ & $22,5 \pm 5,9$ & $18,8 \pm 4,7$ & $14,7 \pm 4,9$ & $\begin{array}{c}11,3 \pm 4,5 \\
* * *\end{array}$ & $97,6 \pm 25,4$ \\
\hline $\mathrm{R} 6+\mathrm{R} 16$ & $64,3 \pm 5,5$ & $66,6 \pm 5,6$ & $45,7 \pm 4,1$ & $50,9 \pm 4,4$ & $43,1 \pm 4,1$ & $270,0 \pm 15,7$ \\
\hline
\end{tabular}

1 R6 : période après ingestion d'un repas (régime à $6 \%$ de cellulose); R16 : période après ingestion d'un repas (régime à $16 \%$ de cellulose); J6 : période de maintien du jeûne après accoutumance au régime R6; J16: période de maintien du jê̂ne après accoutumance au régime R16. Signification statistique : dans une même colonne, comparaison J-R: ${ }^{*} P<0,01,{ }^{*} P<0,05$. Comparaison J6-J16, et comparaison R6-R16 : les moyennes avec un exposant différent sont significativement différentes: majuscules $P<0,05$, minuscules $P<0,01$. Les différences dues au taux de cellulose pour l'ensemble des données $(J 6+R G v s R 6+R 16)$ ne sont significatives $(P<0.05)$ qu'aux $2^{e}$ et $4^{e}$ $\mathrm{h}$, et pour la période totale. ${ }^{2}$ Quantités ingérées $(\mathrm{g}$ ) dans un repas de $800 \mathrm{~g}$. Glucides non cellulosiques : R6:535, R16 = 471; glucose (sur la base de $180 \mathrm{~g}$ pour $162 \mathrm{~g}$ d'amidon) : R6 594, R16 523.

Tableau IV. Évolution des quantités d'azote aminé (en g) apparaissant dans le sang portal au cours de la période post-prandiale $(5 \mathrm{~h})$ ou du maintien du jeûne selon le régime 1,2 .

\begin{tabular}{|c|c|c|c|c|c|c|}
\hline & Inten & alles de temp & depuis le débc & It de la périod & d'observation & \\
\hline & $0-1$ & $1-2$ & $2-3$ & $3-4$ & $4-5$ & $0-5$ \\
\hline $56^{1}$ & $1,12 £ 0,37$ & $1,15 \pm 0,28$ & $1,14 \pm 0,32^{\mathrm{a}}$ & $1,13 \pm 0,39$ & $0,78 \pm 0,41$ & $5,32 \pm 1,67$ \\
\hline R6 1 & $1,77 \pm 0,45$ & $1,94 \pm 0,38$ & $2,46 \pm 0,74$ & $2,56 \pm 0,52^{a}$ & $1,36 \pm 0,56$ & $10,09 \pm 2,34$ \\
\hline $\mathrm{J} 16^{1}$ & $1,01 \pm 0,39$ & $0,63 \pm 0,16$ & $0,79 \pm 0,28^{\circ}$ & $0,66 \pm 0,36$ & $0,57 \pm 0,28^{*}$ & $3,69 \pm 1,27$ \\
\hline R16 1 & $1,61 \pm 0,15$ & $2,29 \pm 0,44$ & $1,76 \pm 0,24$ & $1,56 \pm 0,43^{b}$ & $1,83 \pm 0,34$ & $9,04 \pm 1,06$ \\
\hline$\sqrt{6}+\sqrt{16}$ & $\begin{array}{c}1,07 \pm 0,25 \\
*\end{array}$ & $\underset{* *}{0,89 \pm 0,18}$ & $0,97 \pm 0,21$ & $\begin{array}{c}0,89 \pm 0,26 \\
\ldots\end{array}$ & $\begin{array}{c}0,68 \pm 0,24 \\
\ldots\end{array}$ & $\underset{* \star *}{4,51 \pm 1,03}$ \\
\hline$R 6+R 16$ & $1,69 \pm 0,23$ & $2,11 \pm 0,28$ & $2,11 \pm 0,39$ & $20,6 \pm 0,36$ & $1,60 \pm 0,32$ & $9,57 \pm 1,22$ \\
\hline
\end{tabular}

\footnotetext{
1 R6 : période après Ingestion d'un repas (régime à $6 \%$ de cellulose); R16 : période après ingestion d'un repas (régime à $16 \%$ de cellulose); J6: période de maintien du jeûne après accoutumance au régime R6: J16: période de maintien du jeûne après accoutumance au régime $R 16$. Signification statistique : dans une même colonne, comparaison J-R: " $P<0,01, " P<0,05$. Comparaison J6-J16, et comparaison R6-R16: les moyennes avec un exposant différent sont significativement différentes : minuscules $P<0.01$. Les différences dues au taux de cellulose pour l'ensemble des données ( $\mathrm{J} 6+\mathrm{R} 6$ vs $\mathrm{J} 16+\mathrm{R} 16)$ ne sont significatives $(P<0,05)$ qu'à la $4{ }^{\mathrm{e}} \mathrm{h} .{ }^{2}$ Quantités ingérées $(\mathrm{g})$ dans un repas de $800 \mathrm{~g}$. Azote aminé : R6 : 15,71, R16:16,31.
} 
régime à faible taux de cellulose que dans le cas du régime à fort taux de cellulose (NS, $P<0,18$ pour la période $0-5 \mathrm{~h}$ ), les différences n'étant significatives $(P<0,01)$ qu'à la $3^{\mathrm{e}} \mathrm{h}$. Durant la période postprandiale, les quantités d'azote aminé absorbées sont équivalentes quel que soit le régime, représentant en $5 \mathrm{~h}$ respectivement $63,9 \%$ (R6) et $55,5 \%$ (R16) des quantités ingérées. Les quantités horaires maximales sont enregistrées après $2 \mathrm{~h}$ (R16) ou $4 \mathrm{~h}$ (R6), une différence significative $(P<0,01)$ existant à cette période entre les 2 régimes. Ces quantités sont plus élevées que celles enregistrées pendant le maintien du jeûne, à la $3^{\mathrm{e}} \mathrm{h}$ (R6), à la $2^{\mathrm{e}} \mathrm{h}(\mathrm{R} 16)$, et pour l'ensemble de la période. Ces différences sont plus marquées $(P<0,01$ pour chaque tranche horaire) lorsque sont comparées les données d'ensemble des animaux à jeun $(\mathrm{J} 6+\mathrm{J} 16)$ à celles des animaux nourris ( $R 6+R 16)$.

Les quantités d'acide L-lactique apparues dans le sang portal (tableau $V$ ) lors du maintien au jeûne sont identiques quel que soit le régime reçu antérieurement. Après le repas, il apparaît dans le sang portal de fortes quantités d'acide Llactique, plus élevées au cours des 2 premières heures que par la suite. Ces quantités ont tendance à être plus élevées $(P<$ 0,06 pour l'ensemble de la période) après ingestion du régime à taux modéré de cellulose (R6) qu'après ingestion du régime à taux élevé de cellulose ( $P<0,05$ à la $4 \mathrm{e} h$ ). Elles représentent environ 3 fois les quantités apparues durant la période de jeûne (différences significatives). Les quantités d'acide D-lactique apparues dans la veine porte sont beaucoup plus faibles (16 à $26 \%$ des quantités d'acide L-lactique apparues pendant le jeûne en 5 h, 7 à $13 \%$ des quantités apparues après le repas). Elles sont plus élevées $(P<0,05)$ après le repas $(R 6+R 16: 1,86 \pm 0,32$ g) que lors du maintien du jeûne $(\mathrm{J} 6+\mathrm{J} 16: 1,30 \pm$ $0,26 \mathrm{~g}$ ), et lorsque les animaux sont accou- tumés à un régime à taux élevé de cellulose (J16 + R16: 1,97 $\pm 0,39 \mathrm{~g})$, comparé au régime à taux faible de cellulose ( $\mathrm{J} 6+$ R6 : $1,19 \pm 0,27 \mathrm{~g}$ ).

\section{Consommation d'oxygène}

Quel que soit le traitement (repas vs jeûne, R6 vs R16), la concentration sanguine d'oxygène décroît de 10 à $13 \%$ entre $t_{0}$, temps du début de la période d'observation et $t_{0}+300 \mathrm{~min}$, la concentration d'hématocrite diminuant simultanément de $9 \%$. La concentration d'oxygène est toujours très significativement $(P<0,001)$ plus élevée dans le sang artériel que dans le sang portal (fig 2). La moyenne des concentrations artérielles (mmol/l) pour l'ensemble de la période est $4 \%$ plus élevée après le repas que pendant le maintien au jeûne (R6 5,63 $\pm 0,27, n=5$ vs J6 5,46 $\pm 0,31$, $n=5, P<0,07 ; \mathrm{R} 165,60 \pm 0,13 n=5$ vs J16 5,33 $\pm 0,16 n=5, P<0,005)$. La comparaison entre animaux nourris et animaux à jeun pour l'ensemble des données (R6 + R16 vs J6 + J16) fait ressortir des différences significatives $(P<0,05$ à $P<0,01)$ entre les concentrations artérielles à toutes les périodes d'enregistrement sauf $t_{0}, t_{0}+$ 150 , et $t_{0}+300$. Chez les animaux maintenus au jeûne, la différence porto-artérielle de concentration d'oxygène reste invariable en fonction du temps; les moyennes enregistrées sur la totalité de la période de jeûne étant identiques ( $\mathrm{J} 6: 1,91 \pm 0,03$ $\mathrm{mmol} / \mathrm{l}, n=10 ; \mathrm{J} 16: 1,94 \pm 0,02 \mathrm{mmol} / \mathrm{l}$, $n=10$ ) quel que soit le régime auquel ils ont été accoutumés. Chez les animaux ayant reçu un repas, la différence portoartérielle de concentration d'oxygène augmente dès la prise du repas et culmine à $90 \mathrm{~min}$ (R6 : $10 \%$ ) ou à $120 \mathrm{~min}$ (R16 : $13 \%)$, cette élévation persistant jusqu'à la $4^{e} \mathrm{~h}$ (R6) ou à la $5^{\mathrm{e}} \mathrm{h}$ (R16). Sur l'ensemble de la période d'observation, les moyennes de ces différences porto- 


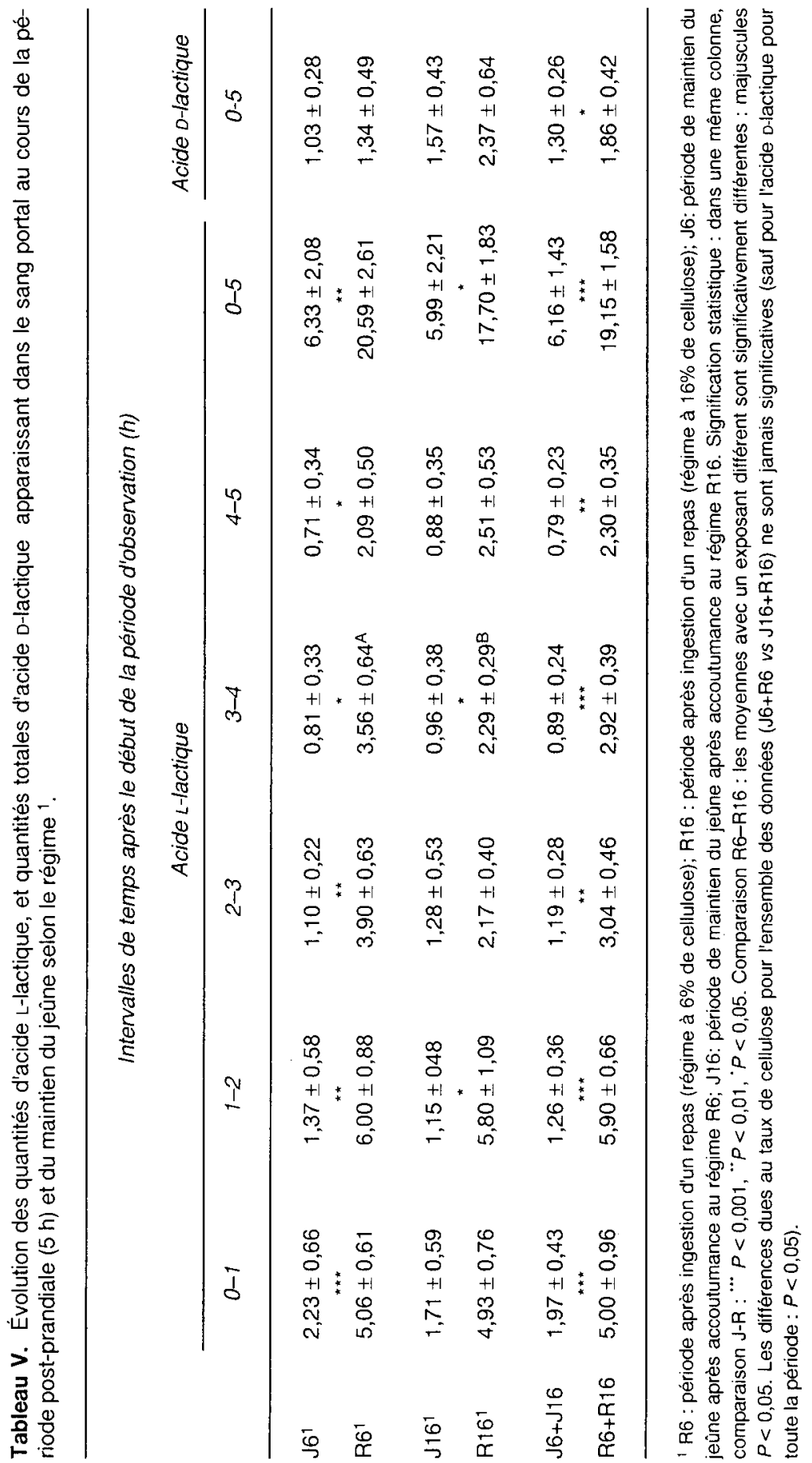




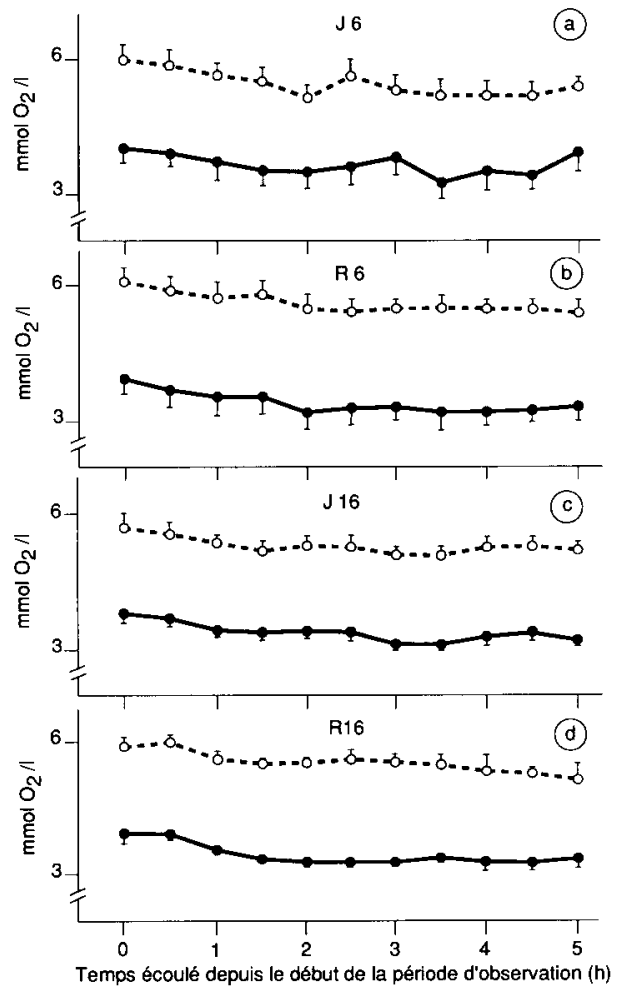

Fig 2. Évolution des concentrations artérielles (0---o) et portales (.-•) d'oxygène ( $\mathrm{mmol} / \mathrm{l})$. a). Pendant une période de maintien du jeûne (J6) débutant $16 \mathrm{~h}$ après le dernier repas $(800 \mathrm{~g})$ de régime à taux modéré de cellulose. b). Pendant la période post-prandiale après un repas $(800 \mathrm{~g})$ d'un régime à taux modéré de cellulose (R6). c). Pendant une période de maintien du jeûne débutant $16 \mathrm{~h}$ après le dernier repas (800 g) d'un régime à taux élevé de cellulose. d). Pendant la période post-prandiale après un repas (800 g) d'un régime à taux élevé de cellulose (R16). Chaque point représente la moyenne (et l'écart type de la moyenne de 5 données. Les écarts entre concentrations artérielles et portales sont hautement significatifs $(P<0,001)$.

artérielles ne sont pas significativement différentes, quel que soit le régime ingéré (R6 : 2,20 $\pm 0,03 \mathrm{mmol} / \mathrm{l}, n=10$; R16 : $2,13 \pm 0,03 \mathrm{mmol} / \mathrm{l}, n=10$, NS). Compte tenu de ces similitudes, il est possible de regrouper les valeurs post-prandiales obte-

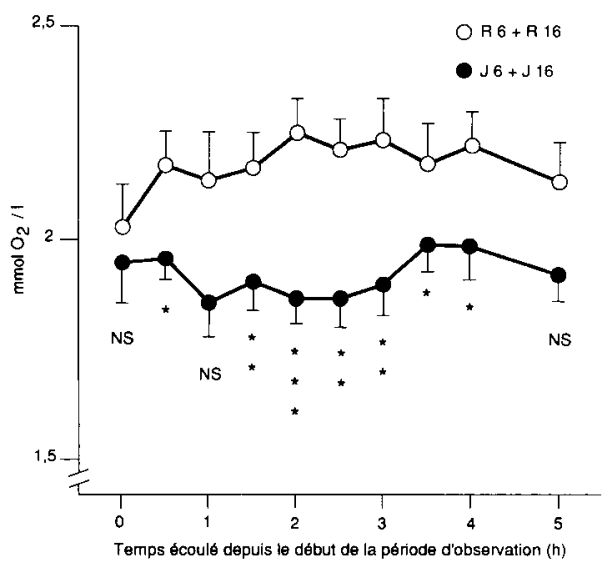

Fig 3. Évolution des différences artério-portales $\left.\left(A_{(02)}\right)^{-} P_{(02)}\right)$ de concentration d'oxygène (mmol/l). - - pendant la période de maintien du jeûne débutant $16 \mathrm{~h}$ après le dernier repas $(800 \mathrm{~g})$; $\mathrm{o} \longrightarrow$ o pendant la période post-prandiale après ingestion d'un repas $(800 \mathrm{~g})$. Chaque point représente la moyenne (les barres verticales représentant l'écart type de la moyenne) de 10 données recueillies chez des animaux soumis au régime $\mathrm{R} 6$ (à taux modéré de cellulose, $n=$ 5) et au régime R16 (à taux élevé de cellulose, $n=5)$ ou à des périodes de jeûne correspondant (J6, $n=5 ;$ J16, $n=5$ ). Signification statistique : ${ }^{\star \star \star} P<0,001 ;{ }^{\star \star} P<0,01 ;{ }^{*} P<0,05$.

nues après ingestion des 2 régimes et de les comparer aux valeurs obtenues chez les animaux à jeun (fig 3). Dans ces conditions, les différences porto-artérielles sont à tout moment (sauf aux moments $t_{0}, t_{0}+$ $1 \mathrm{~h}$, et $\left.t_{0}+5 \mathrm{~h}\right)$ significativement $(P<0,05$ à 0,01 ) plus grandes au cours de la période post-prandiale, qu'au cours du jeûne correspondant.

En raison de la diminution de concentration artérielle d'oxygène avec le temps écoulé après le repas, la quantité d'oxygène disponible décroît avec le temps (tableau VI), la diminution totale s'élevant entre 7 et $10 \%(\mathrm{~J} 6+\mathrm{J} 16: P<0,05 ; \mathrm{R} 6+$ R16 : $P<0,01)$. Pour chacun des régimes, les quantités d'oxygène disponibles, qu'elles soient horaires ou cumulées sur l'ensemble de la période d'observation, ont 


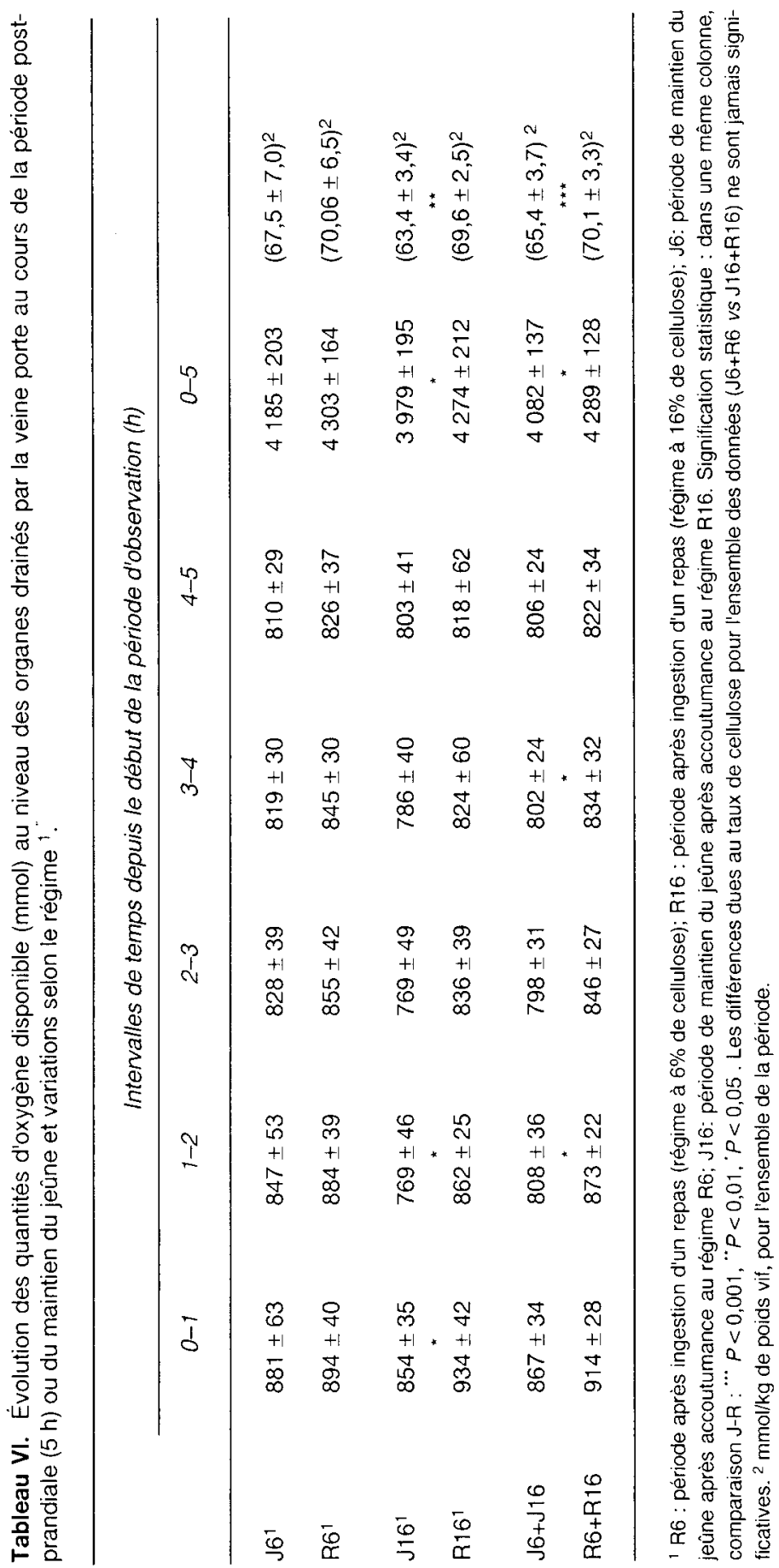


tendance à être plus élevées après ingestion d'un repas qu'au cours du maintien du jeûne. Comparant l'ensemble des données obtenues chez les animaux nourris (R6 et $R 16)$ à celles obtenues chez ceux maintenus au jeûne $(\mathrm{J} 6+\mathrm{J} 16)$, il s'avère que la $\mathrm{D}\left(\mathrm{O}_{2}\right)$ a été accrue par la prise alimentaire, aussi bien pour la période totale d'observation $(P<0,05)$ que pour certains intervalles horaires ( $2^{e}$ et $4^{e} h$ ). Par contre, la quantité d'oxygène disponible n'est pas modifiée par la nature du régime. II n'existe aucune relation entre la disponibilité de l'oxygène et le poids vif des animaux.

La consommation d'oxygène reste invariable au cours du maintien du jeûne, quel que soit le régime antérieur. Elle s'élève dès la prise de nourriture pour culminer entre la $2^{\mathrm{e}}$ et $4^{\mathrm{e}} \mathrm{h}$ après ingestion de $\mathrm{R} 6$, et à la $3^{\mathrm{e}} \mathrm{h}$ après ingestion de R16 (tableau VII). Elle devient ainsi significativement plus élevée $(P<0,05)$ chez les animaux nourris que chez ceux maintenus au jeûne pendant ces intervalles et pour la totalité de la période d'observation. Considérant l'ensemble des 2 régimes, les différences de consommation d'oxygène entre prise de repas et maintien du jeûne sont significatives $(P<0,05$ à $P<0,01)$ de la $2^{\mathrm{e}}$ à la $4^{\mathrm{e}} \mathrm{h}$ de la période d'observation et très significatives $(P<0,01)$ pour la totalité de cette période. Par contre, il n'existe aucune différence de consommation d'oxygène en fonction du régime. La consommation d'oxygène est en relation $(r=0,56)$ significative $(P<0,01 ; n=20)$ avec le poids vif de l'animal.

Chez les animaux accoutumés au régime à $6 \%$ de cellulose, le coefficient d'utilisation de l'oxygène a tendance à être plus élevé lors de la période post-prandiale que lors du maintien au jeûne (ensemble de la période $P<0,12$ ), mais la différence n'est significative qu'à la $3^{\mathrm{e}} \mathrm{h}$ (tableau VIII). Considérant l'ensemble des données (R6 $+\mathrm{R} 16$ vs $\mathrm{J} 6+\mathrm{J} 16)$, on retrouve la même tendance $(P<0,06$ pour la totalité de la période), mais la différence n'est significative $(P<0,05)$ qu'à la $3^{3} \mathrm{~h}$. Le coefficient d'utilisation de l'oxygène reste invariable lors du maintien du jeûne, et augmente après ingestion du repas $\left(P<0,05\right.$ au $2^{e}$ et $4^{\mathrm{e}} \mathrm{h}, P<0,001$ à la $\left.3^{\mathrm{e}} \mathrm{h}\right)$. Ce coefficient est identique pour les 2 types de régime.

\section{Production de chaleur}

Sur la base d'une production de chaleur de 20,4 kJ/l d'oxygène (Blaxter, 1962), ce qui correspond à 109,3 kcal/mol d'oxygène, la production de chaleur des organes drainés par la veine porte est significativement plus élevée après la prise d'aliment qu'au cours du maintien du jeûne, que le taux de cellulose du régime soit modéré (R6 : 37,8 $\mathrm{kcal} / \mathrm{h} ; \mathrm{J} 6: 31,7 \mathrm{kcal} / \mathrm{h}, P<0,05)$ ou élevé (R16 : 35,7 kcal/h; J16 : 32,2 kcal/h, $P<$ $0,05)$.

\section{DISCUSSION}

Le débit portal enregistré dans la présente expérience présente quelques divergences avec celui enregistré par d'autres auteurs. Ainsi, dans la présente expérience $(40,0$ à $41,7 \mathrm{ml} / \mathrm{kg} / \mathrm{min})$, il est plus élevé que celui $(34,5 \mathrm{ml} / \mathrm{kg} / \mathrm{h})$ enregistré par Yen et al (1989) chez des porcs en croissance placés au jeûne depuis $24 \mathrm{~h}$. En outre, il ne se produit qu'une faible augmentation de ce débit $(4,5 \%)$ au cours de la période post-prandiale, alors que cette augmentation peut atteindre $46 \%$ selon Yen et al (1989), et $35 \%$ selon Nowicki et al (1983) chez le porcelet nouveau-né. Ces différences peuvent trouver leur origine dans les conditions expérimentales, notamment par la durée du jeûne préprandial ( $24 \mathrm{~h}$ dans le cas des animaux de Yen et al, 1989 , vs $16 \mathrm{~h}$ dans la présente expérience) et par le volume du repas ingéré 


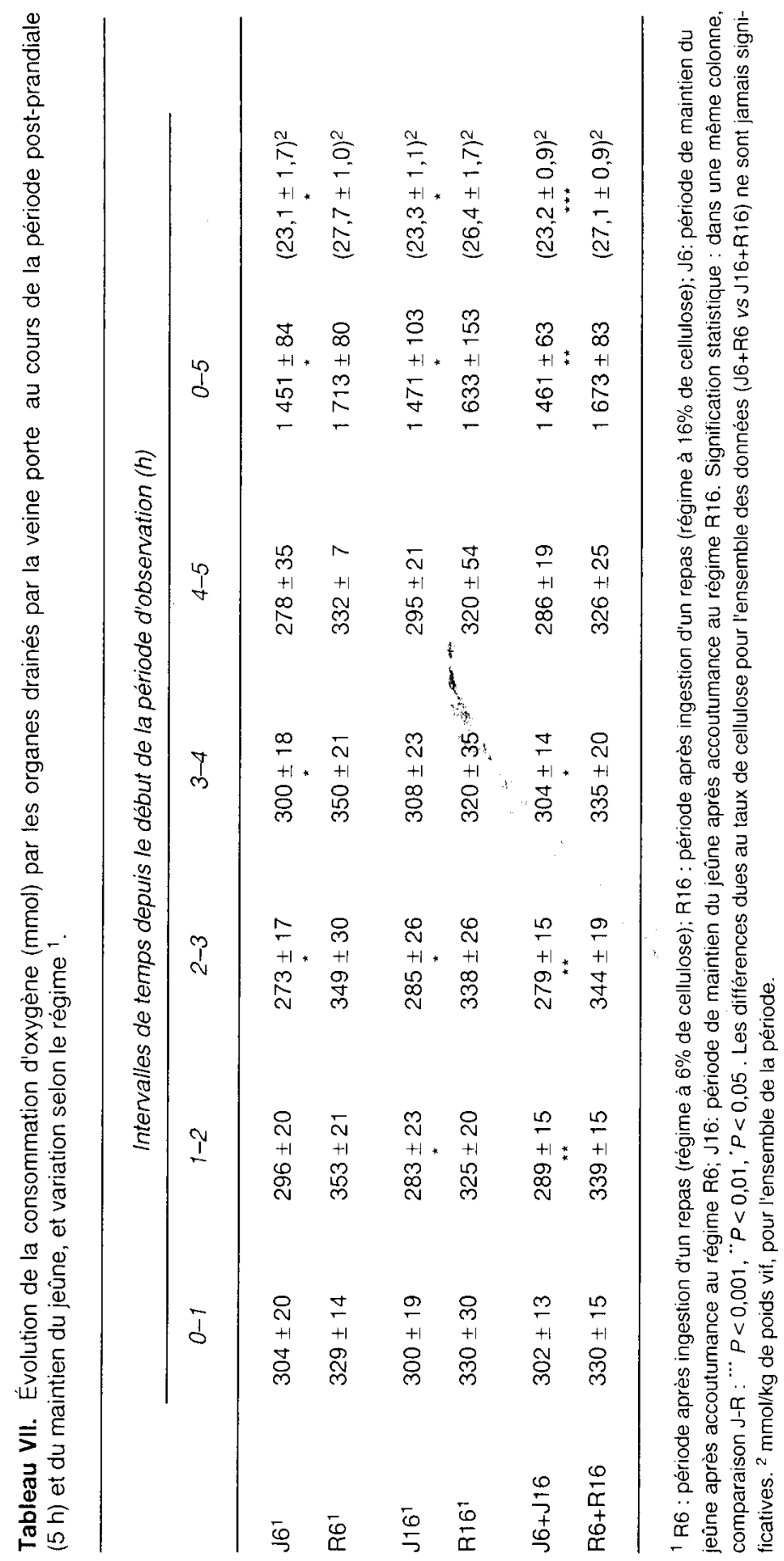


Tableau VIII. Évolution du coefficient d'utilisation de l'oxygène (\%) $)^{1}$ par les organes drainés par la veine porte au cours de la période post-prandiale $(5 \mathrm{~h})$ et du maintien du jeûne et variation selon le régime ${ }^{2}$.

Intervalles de temps depuis le début de la période d'obsenvation $(5 h)$

$\begin{array}{llllll}0-1 & 1-2 & 2-3 & 3-4 & 4-5 & 0-5\end{array}$

$\begin{array}{lllllll}\text { J62 } & 35,0 \pm 2,5 & 35,3 \pm 2,8 & 33,2 \pm 2,5 & 36,8 \pm 2,6 & 34,3 \pm 4,3 & 34,9 \pm 2,4 \\ \text { R62 } & 37,1 \pm 2,3 & 40,5 \pm 3,6 & 41,1 \pm 3,6 & 41,8 \pm 3,5 & 40,6 \pm 2,6 & 40,2 \pm 2,9 \\ \text { J162 } & 35,1 \pm 1,3 & 36,6 \pm 1,1 & 38,0 \pm 1,9 & 38,4 \pm 0,7 & 36,6 \pm 1,4 & 36,8 \pm 1,1 \\ \text { R16 } & 35,1 \pm 1,8 & 37,6 \pm 1,7 & 40,3 \pm 1,5 & 38,4 \pm 1,5 & 37,7 \pm 4,1 & 38,1 \pm 1,9 \\ \text { J6+J16 } & 35,0 \pm 1,3 & 35,9 \pm 1,4 & 35,6 \pm 1,7 & 37,6 \pm 1,3 & 35,5 \pm 2,2 & 35,9 \pm 1,3 \\ \text { R6+R16 } & 36,1 \pm 1,4 & 39,1 \pm 2,0 & 40,7 \pm 1,8 & 40,1 \pm 1,9 & 39,1 \pm 2,4 & 39,1 \pm 1,7\end{array}$

\footnotetext{
1 Rapport de la consommation d'oxygène à la quantité d'oxygène disponible; ${ }^{2}$ R6 : période après ingestion d'un repas (régime à $6 \%$ de cellulose); R16 : période après ingestion d'un repas (régime à $16 \%$ de cellulose); J6: période de maintien du jeûne après accoutumance au régime R6; J16: période de maintien du jeûne après accoutumance au régime R16. Signification statistique : dans une mème colonne, comparaison J-R : ${ }^{\prime} P<0,01$. Les différences dues au taux de cellulose pour l'ensemble des données (J6+R6 vs J16+R16) ne sont jamais significatives.
}

(1 $200 \mathrm{~g}$ pour Yen et al, 1989; $800 \mathrm{~g}$ dans la présente expérience). Or, on sait que la digestion d'un repas s'étend sur une période plus ou moins longue selon la quantité ingérée, cette période pouvant atteindre 20 à $22 \mathrm{~h}$ pour les glucides et 15 à $18 \mathrm{~h}$ pour les protéines (Rérat, 1981). De ce fait, $16 \mathrm{~h}$ après un repas de $800 \mathrm{~g}$, il peut subsister des résidus importants d'aliments dans l'intestin grêle et une activité digestive importante impliquant le maintien d'un débit sanguin élevé. L'arrivée de nouveaux aliments dans un tube digestif non encore complètement évacué pourrait ainsi ne pas provoquer une hyperémie aussi marquée que lors d'un jeûne plus long. Par ailleurs, le niveau d'ingestion d'énergie métabolisable est en relation avec le débit portal chez le mouton (Webster et al, 1975) et on peut concevoir qu'un repas moins important chez le porc puisse provoquer une plus faible augmentation du débit portal qu'un repas de faible niveau.

La présence de résidus du repas antérieur dans le tube digestif est confirmée par l'absorption de quantités notables de glucose et d'azote aminé au cours du jeûne. Surtout dans les premières heures, les quantités apparues dans la veine porte, au cours du maintien du jeûne, sans être importantes, sont loin d'être négligeables. En outre, il résulte de la digestion de ces résidus que les quantités absorbées après ingestion du repas sont, pour un même intervalle de temps, supérieures de 30 à $40 \%$ à celles mesurées dans une expérience antérieure (Giusi-Périer et al, 1989) dans laquelle les mêmes régimes, $R 6$ et 
R16, étaient utilisés mais après un jeûne de $20 \mathrm{~h}$. Comme dans cette expérience, toutefois, l'élévation du taux de cellulose se traduit par une diminution de l'absorption de glucose (significative pour l'ensemble de la période et pour l'intervalle 3$4 \mathrm{~h})$ mais non significative en termes de coefficient d'absorption en raison des différences de niveau d'ingestion de l'amidon. Elle se traduit également par une diminution de l'absorption d'azote aminé, seulement significative entre les $3^{e}$ et $4^{e} \mathrm{~h}$. La depression de l'absorption des nutriments azotés due à l'augmentation de l'apport cellulosique est en accord avec ce qui est démontré par les études de digestibilité iléale, et les mécanismes de cette dépression ont été souvent analysés (Rérat, 1992).

Le métabolisme de l'entérocyte et des micro-organismes intestinaux est à l'origine de la formation des acides $D$ et Llactiques. La forme $D$ représente en principe la moitié environ du DL lactate produit par les micro-organismes (Giesecke et Stangassinger, 1980) alors que le métabolisme anaérobie des cellules de la paroi digestive ne fournit que du L-lactate. Lors du maintien du jeûne, les quantités de Llactate formées sont généralement peu importantes mais persistantes, ce qui signe une activité cellulaire notable, et plus élevées en début de période que par la suite, en raison sans doute de l'existence de résidus du régime antérieur dans la lumière digestive. Les quantités sont plus élevées lors de la période post-prandiale que lors du maintien au jeûne, en raison de l'activité accrue de la paroi digestive. Les quantités apparues au cours de la période postprandiale sont analogues à celles mesurées antérieurement avec des régimes à base d'amidon de maïs (Rérat et al, 1984). Comme dans une expérience antérieure utilisant les mêmes régimes (Giusi-Périer et al, 1989), on constate une tendance à plus forte absorption de L-lactate lors de l'ingestion du régime à taux modéré de cellulose (R6) qu'après ingestion d'un régime à taux élevé de cellulose (R16), sans que cette différence deviennne significative, sauf à la $4 \mathrm{e} \mathrm{h}$.

Pendant le maintien du jeûne, l'absorption d'acide $D$-lactique est inférieure $(P<$ 0,05 ) à celle mesurée au cours de la période post-prandiale pour les 2 régimes. Elle représente alors 16,3\% (J6) à 26,2\% (J16) de l'absorption de l'acide L-lactique, ce qui montre l'importance relative élevée de l'activité microbienne durant cette période. Par contraste, durant la période post-prandiale, l'absorption de l'acide Dlactique ne représente plus que 6,5\% (R6) et $13,4 \%$ (R16) de l'acide L-lactique, pourcentages analogues à ceux mesurés dans une expérience antérieure avec un régime à base de maltose (Rérat et al, 1993).

Les concentrations artérielles et portales d'oxygène sont analogues à celles trouvées par d'autres auteurs chez le porcelet (Nowicki et al, 1983) et l'agneau (Edelstone et Holzman, 1981). Que ce soit après l'ingestion d'un repas ou le maintien du jeûne, pendant une période de $5 \mathrm{~h}$ débutant $16 \mathrm{~h}$ après le repas, la concentration artérielle d'oxygène décroît progressivement de 10 à $13 \%$ au cours de cette période. Cette diminution est analogue à celle enregistrée chez le porcelet nouveauné (Nowicki et al, 1983). En raison, de la diminution de concentration d'oxygène, et de la faible modification de débit sanguin, la $\mathrm{D}\left(\mathrm{O}_{2}\right)$ décroît aussi bien après le repas que lors du maintien au jeûne au-delà de $16 \mathrm{~h}$. Pour la période post-prandiale, ces résultats divergent de ceux des auteurs déjà cités (Nowicki et al, 1983; Edelstone et Holzman, 1981) pour lesquels la $\mathrm{D}\left(\mathrm{O}_{2}\right)$ augmente transitoirement après le repas en raison d'une augmentation du débit de sang dans les organes digestifs. À noter que la disponibilité de l'oxygène serait beaucoup plus élevée tant à jeun qu'après le repas chez le porcelet nouveau-né (Nowicki et al, 1983) en raison du débit portal 
relativement beaucoup plus important que celui enregistré dans la présente expérience. Quoi qu'il en soit, à l'instar de ce qui est trouvé par les autres auteurs (Nowicki et al, 1983; Edelstone et Holzman, 1981), la disponibilité de l'oxygène dans la présente expérience est plus élevée pendant la période post-prandiale que pendant le maintien du jeûne. L'augmentation de disponibilité est cependant faible, et provient, d'une part, d'une concentration artérielle d'oxygène plus élevée $(3 \%)$, d'autre part, d'une élévation du débit portal, certes faible, mais plus marquée durant la période post-prandiale que pendant le maintien du jeûne.

La consommation d'oxygène pendant la période de maintien du jeûne (J6:1,72 ml/ $\mathrm{min} / \mathrm{kg} ; \mathrm{J} 16: 1,74 \mathrm{ml} / \mathrm{min} / \mathrm{kg}$ poids vif) est légèrement supérieure (13\%) à celle enregistrée (Yen et al, 1989) chez des porcs de 4 mois après un jeûne de $24 \mathrm{~h}(1,52 \mathrm{ml})$ $\mathrm{min} / \mathrm{kg}$ poids vif) en raison sans doute de la présence de reliquats du repas de la veille dans le tube digestif. Elle est significativement plus élevée de la $2^{e}$ à la $4^{e} \mathrm{~h}$ après l'ingestion d'un repas que pendant la période de maintien de jeûne, sans que l'augmentation moyenne soit marquée de façon plus significative pour le régime à $6 \%$ de cellulose que pour le régime à $16 \%$ de cellulose (R6 : 20\%; R16 : 13\%). Cette augmentation liée au repas confirme les résultats de Yen et al (1989) même si l'accroissement n'est pas aussi marqué que celui $(46 \%)$ enregistré par ces auteurs. Les différences sont liées aux conditions expérimentales différentes, quant au niveau d'ingestion et à la durée du jeûne préprandial. On sait (Webster et al, 1975) que la consommation d'oxygène est en relation directe avec le niveau d'ingestion chez le mouton. Or, dans la présente expérience, le niveau d'ingestion est sensiblement plus faible et la durée du jeûne préprandial beaucoup plus courte que celles des animeaux mis en expérience par Yen et al
(1989), ce qui peut expliquer la moindre consommation d'oxygène. L'élévation postprandiale de la consommation d'oxygène a été également signalée par d'autres auteurs (Nowicki et al, 1983; Edelstone et Holzman, 1981). Enfin, la consommation d'oxygène n'est pas influencée par le taux de cellulose du régime, malgré l'influence connue de la cellulose sur le péristaltisme de l'intestin, les sécrétions digestives et la multiplication des entérocytes (Rérat, 1992).

L'extraction d'oxygène, tant pendant la période de maintien du jeûne (35 à $37 \%$ ) qu'après ingestion d'un repas de $800 \mathrm{~g}$ (38 à $40 \%$ ) est beaucoup plus élevée que celle enregistrée chez le porcelet nouveau-né (20 à $25 \%$ selon Nowicki et al, 1983) ou l'agneau nouveau-né (28\% selon Edelstone et Holzman, 1981). Ce fait est liè à la très forte $\mathrm{D}\left(\mathrm{O}_{2}\right)$ mesurée par ces auteurs en raison d'un débit sanguin très élevé dans les organes digestifs. L'extraction d'oxygène n'est pas augmentée de façon significative par la prise alimentaire (R6 : 15,2\% ; R16 : 3,6\%) dans la présente expérience, alors qu'elle est augmentée de $33 \%$ chez le porcelet nouveau-né (Nowicki et al, 1983) et de $45 \%$ chez l'agneau nouveau-né (Edelstone et Holzman, 1981). Cette différence s'explique par les niveaux initiaux du coefficient d'extraction, déjà très élevés dans la présente expérience, et très faibles dans les expériences des auteurs cités. Enfin, le taux de cellulose du régime n'a aucune influence sur l'extraction d'oxygène par les organes digestifs.

La dépense énergétique des organes drainés par la veine porte peut être calculée à partir de la consommation d'oxygène (métabolisme aérobie) et la production d'acide lactique (métabolisme anaérobie). Pendant le maintien au jeûne, la fraction provenant du métabolisme aérobie ( $\mathrm{J} 6$ : $31,7 \mathrm{kcal} / \mathrm{h} ; \mathrm{J} 16: 32,2 \mathrm{kcal} / \mathrm{h}$ ) est 2 fois plus élevée que celle enregistrée chez le mouton (environ $15 \mathrm{kcal} / \mathrm{h}$ ) après $48 \mathrm{~h}$ de 
jeûne (Webster et al, 1975). Elle augmente de 13 à $20 \%$ après le repas, ce qui se traduit par une dépense énergétique supplémentaire de $3,6 \mathrm{kcal} / \mathrm{h}$ (R16) à 6,1 $\mathrm{kcal} / \mathrm{h}$ (R6), correspondant à 1,2-2,1 kcal/ $1000 \mathrm{kcal}$ d'ingéré, ces valeurs étant du même ordre de grandeur que celles fournies par Webster et al (1975). Si I'on se base sur un changement d'enthalpie de $11,1 \mathrm{kcal}$ environ pour $1 \mathrm{~mol}$ de lactate formée (soit $0,1236 \mathrm{kcal} / \mathrm{g}$ de lactate), la production de lactate d'origine cellulaire à l'exclusion du L-lactate d'origine microbienne ne fournit que $0,48 \mathrm{kcal} / \mathrm{h}$ (R6) ou 0,38 $\mathrm{kcal} / \mathrm{h}$ (R16); pendant le maintien du jeûne, ces valeurs tombent à $0,11-0,13$ $\mathrm{kcal} / \mathrm{h}$. Ces valeurs ne représentent que $0,3-0,4 \%$ du métabolisme aérobie à jeun et $1,3(\mathrm{R} 6)$ et $1,1 \%$ (R16) durant la période post-prandiale.

On sait que le métabolisme des tissus intestinaux utilise comme substrat énérgétique non seulement le glucose, mais également la glutamine (Windmueller et Spaeth, 1980, Rérat et al, 1988), celle-ci représentant une fraction importante (Windmueller, 1982) de l'énergie consommée. Cette captation de glutamine d'origine artérielle peut s'élever à environ $1 \mathrm{~g} / \mathrm{h}$ (Rérat et al, 1992). Il est donc impossible, dans les conditions de cette expérience, de préciser la quantité de glucose qui a été nécessaire pour couvrir le besoin énergétique. On peut rappeler que, d'après des estimations antérieures, la captation de glucose se situe entre $7,5 \mathrm{~g} / \mathrm{h}$ (Rérat et al, 1990), 14 à $21 \mathrm{~g} / \mathrm{h}$ (Rérat et al, 1984) et 13 à $17 \mathrm{~g} / \mathrm{h}$ (Rérat et al, 1992). Une fraction importante de cette captation est prise en compte pour la formation de lactate : 0,88 à $1,1 \mathrm{~g} / \mathrm{h}$ pendant le maintien du jeûne, 3,1 à $3,8 \mathrm{~g} / \mathrm{h}$ respectivement après ingestion de R16 et de R6. Sachant qu'une molécule d'oxygène permet l'oxydation de $30 \mathrm{mg}$ de glucose, la quantité maximum de glucose métabolisé pour correspondre à la consommation d'oxygène pourrait s'élever à $3,75 \mathrm{~g} / \mathrm{h}$ pendant le jeûne, et à $10,6 \mathrm{~g} / \mathrm{h}$ (R6) ou 9,8 g/h (R16) pendant la période post-prandiale, quantités que l'on peut minorer de 30 à $40 \%$ si l'on tient compte de l'intervention de la glutamine dans le métabolisme énergétique (Windmueller, 1982).

En conclusion, l'ingestion d'un repas de $800 \mathrm{~g}$ provoque, par rapport au jeûne, une élévation de la consommation d'oxygène des organes drainés par la veine porte, associée à une disponibilité plus élevée de l'oxygène dans cette zone. La dépense énergétique due au métabolisme aérobie des organes drainés par la veine porte se situe entre 35,7 et $37,8 \mathrm{kcal} / \mathrm{h}$ après l'ingestion d'un repas, soit 11 à $18 \%$ de plus que la production d'énergie après un jeûne de $16 \mathrm{~h}$. La production de lactate liée au métabolisme anaérobie varie entre 3,1 et $3,8 \mathrm{~g} / \mathrm{h}$ pendant la période post-prandiale, et autour de $1 \mathrm{~g} / \mathrm{h}$ pendant le jeûne. L'augmentation du taux de cellulose ne provoque aucune modification significative de ces diverses variables.

\section{REMERCIEMENTS}

À $\mathrm{P}$ Vaugelade pour son aide technique, à $F$ Cointepas et $G$ Brachet pour les soins apportés aux animaux, et à $J$ Gallé pour la réalisation des illustrations.

\section{RÉFÉRENCES}

Blaxter KL (1962) The energy metabolism in ruminants. Hutchinson Publ, London

Edelstone DI, Holzman IR (1981) Oxygen consumption by the gastrointestinal tract and liver in conscious newborn lambs. Am J Physiol 240, G297-G304

Gawehn K, Bergmeyer HU (1974) D-(-) Lactate. In: Methods of enzymatic analysis (HU Bergmeyer, ed). Academic Press, New York 
Giesecke D, Stangassinger M (1980) Lactic acid metabolism. In: Digestive physiology and metabolism in ruminants ( $Y$ Ruckebush, $P$ Thivend, eds). MTP Press Ltd, Lancaster, UK

Giusi-Périer A, Fiszlewicz M, Rérat A (1989) Influence of diet composition on intestinal volatife fatty acid and nutrient absorption in unanesthetized pigs. J Anim Sci 67, 386-402

Granger HJ, Norris CP (1980) Intrinsic regulation of intestinal oxygenation in the anesthetized dog. Am J Physiol 238, H836-H843

Hill JB, Kessler G (1961) An automated determination of glucose utilizing a glucose oxidaseperoxidase system. J Lab Clin Med 57, 970980

Minaire $Y$, Foucherand F, Studievic C (1965) Adaptation du dosage de l'acide lactique par voie enzymatique à l'autoanalyzer. In: Proc 4th Int Technicon Symp. Technicon, Domon, France

Nowicki PT, Stonestreet BS, Hansen NB, Yao AC, Oh W (1983) Gastrointestinal blood flow and oxygen consumption in awake newborn piglets. Am J Physiol 245, G697-G702

Palmer DW, Peters R (1965) Simple automatic determination of aminogroups in serum/ plasma using trinitrobenzene sulfonate. In: Automation in analytical chemistry (LT Skeggs, Jr, ed). Technicon Symp, Mediad Inc, New York

Rérat A (1981) Chronologie et bilans de l'absorption des sucres réducteurs et de l'azote aminé chez le porc selon la nature des aliments. Bull Acad Nat Med 165, 1131-1137

Rerat A (1992) Carbohydrate interactions on protein and amino acid digestibility and $a b-$ sorption and metabolic consequences in the pig. In: Proc 6th int symp protein metab and nutr. Herning, Danemark, EAAP publ, $n^{\circ} 59$, I, 37-53

Rérat A, Vaugelade $P$, Villiers $P$ (1980) A new method for measuring the absorption of nutrients in the pigs: critical examination. In : Current concepts of digestion and absorption in pigs (AG Low, IG Partridge, eds). National Institute for Research in Dairying, Reading, UK

Rérat A, Vaissade $P$, Vaugelade $P$ (1984) Absorption kinetics of some carbohydrates in conscious pigs 2. Quantitative aspects. $\mathrm{Br} \mathrm{J}$ Nutr 51, 517-529
Rérat A, Chayvialle A, Kande J, Vaissade $P$, Vaugelade P, Bourrier P (1985) Metabolic and hormonal effects of test meals with various protein contents in pigs. Can J Anim Sci 63, 1547-1559

Rérat A, Simoes Nunes C, Mendy F, Roger L (1988) Amino-acid absorption and production of pancreatic hormones in non-anaesthetized pigs after duodenal infusions of milk enzymic hydrolysate or free amino-acids. Br J Nutr 60, 121-136

Rérat A, Vaissade P, Vaugelade $P(1990)$ Kinetics and balance of glucose and galactose appearance in the portal blood after intake of lactose or hydrolysed lactose in conscious pigs. Ann Nutr Metab 34, 119-132

Rérat $A$, Simoes Nunes $C$, Mendy F, Vaissade $P$, Vaugelade $P$ (1991) Splanchnic fluxes of amino-acids after duodenal infusion of carbohydrate solutions containing free amino-acids or oligopeptides in the non-anaesthetized pig. Br J Nutr $68,111-138$

Rèrat A, Giusi-Périer A, Vaissade P (1993) Absorption balances and kinetics of nutrient and bacterial metabolites in conscious pigs after intake of maltose or maltitol-rich diets. J Anim Sci (sous presse)

Snedecor GW, Cochran WG (1967) Statistical methods. lowa State Univ Press Publ, Ames, IA

Vaugelade $P$, Rérat A (1991) Influence de la prise alimentaire sur la consommation d'oxygène de l'aire splanchnique extra-hépathique chez le porc éveillé. Reprod Nutr Dev 31 , 290

Webster AJT, Osuji PO, White F, Ingram JF (1975) The influence of food intake on portal blood flow and heat production in the digestive tract of sheep. Br J Nutr 34, 125-139

Windmueller HG (1982) Glutamine utilization by the small intestine. Adv Enzymol 53, 201-238

Windmueller HG, Spaeth AE (1980) Respiratory fuels and nitrogen metabolism in vivo in the small intestine of fed rats. Quantitative importance of glutamine, glutamate and aspartate. J Biol Chem 255, 107-112

Yen JT, Nienaber JA, Hill JA, Pond WG (1989) Oxygen consumption by portal vein-drained organs and by whole animal in conscious growing swine. Proc Soc Exptl Biol Med 190, 393-398 